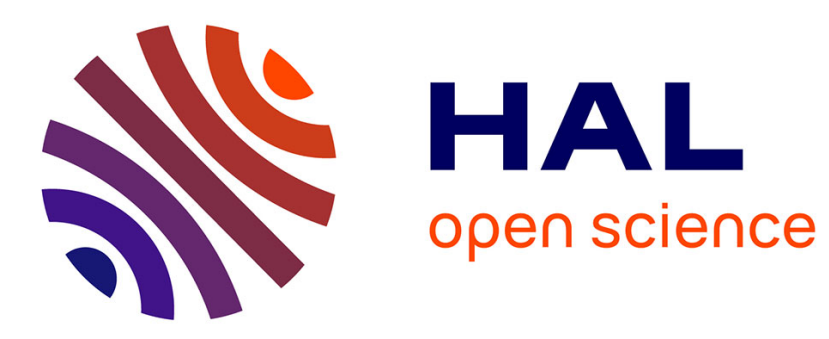

\title{
Changements de phase du gallium à la pression atmosphérique
}

Louis Bosio, André Defrain, Israël Epelboin

\section{To cite this version:}

Louis Bosio, André Defrain, Israël Epelboin. Changements de phase du gallium à la pression atmosphérique. Journal de Physique, 1966, 27 (1-2), pp.61-71. 10.1051/jphys:01966002701-206100 . jpa-00206369

\section{HAL Id: jpa-00206369 https://hal.science/jpa-00206369}

Submitted on 1 Jan 1966

HAL is a multi-disciplinary open access archive for the deposit and dissemination of scientific research documents, whether they are published or not. The documents may come from teaching and research institutions in France or abroad, or from public or private research centers.
L'archive ouverte pluridisciplinaire HAL, est destinée au dépôt et à la diffusion de documents scientifiques de niveau recherche, publiés ou non, émanant des établissements d'enseignement et de recherche français ou étrangers, des laboratoires publics ou privés. 


\title{
CHANGEMENT'S DE PHASE DU GALLIUM A LA PRESSION ATMOSPHÉRIQUE
}

\author{
Par L. BOSIO, A. DEFRAIN et I. EPELBOIN, \\ Laboratoire de Physique des Liquides et d'Électrochimie, \\ Département des Recherches Physiques, Faculté des Sciences, Paris.
}

\begin{abstract}
Résumé. - Après avoir signalé l'importance du choix des échantillons de faible volume dans l'étude de la germination dans le gallium liquide, on montre que les conditions de la germination homogène sont réalisées vers - $123^{\circ} \mathrm{C}$ pour certaines gouttelettes provenant_de l'émulsion du métal liquide ; la phase solide qui apparaît est alors la forme métastable Gaß̉ et l'on mesure l'enthalpie libre interfaciale entre le liquide et un germe de Gaß. Ces échantillons liquides ne cristallisent qu'exceptionnellement en la forme stable $\mathrm{Ga}_{\alpha}$, mais on peut aussi obtenir une deuxième phase solide métastable $\mathrm{Ga}_{\gamma}$ dont on détermine quelques propriétés physiques. Par leurs paramètres thermodynamiques, les formes $\mathrm{Ga}_{\beta}$ et $\mathrm{Ga}_{\gamma}$ sont identifiées respectivement aux formes $\mathrm{Ga} I I$ et Ga $I I I$ stables à haute pression. Des considérations sur la règle des degrés d'Ostwald permettent de justifier l'obtention de ces formes métastables à partin des états liquide ou vapeur.
\end{abstract}

Abstract. -- After pointing out the importance of the choice of small volume samples in the study of nucleation in liquid gallium, the authors show that homogeneous nucleation conditions are obtained at $-123 \mathrm{oC}^{\circ}$ for droplets resulting from the emulsion of the liquid metal. The solid phase which then appears is the metastable form $\mathrm{Ga}_{\beta}$; the free interfacial enthalpy between the liquid and a Ga $\beta$ nucleus is measured. These liquid samples crystallise only exceptionally in the stable form $\mathrm{Ga}_{\alpha}$ but it is possible to obtain a second solid metastable form $\mathrm{Ga}_{\gamma}$ of which some physical characteristics are determined. From their thermodynamic parameters, the forms $\mathrm{Ga}_{\beta}$ and $\mathrm{Ga}_{\gamma}$ are respectively identified with the forms $\mathrm{Ga}$ II and Ga III, stable at high pressure. The production of these metastable forms from liquid or vapour states is proved by the Ostwald degree rule.

Introduction. - La surfusion d'une substance maintenue liquide à une température inférieure à son point de fusion $T_{t}$ cesse avec l'apparition du premier cristal qui donnera naissance à la phase solide. L'expérience montre que la formation du premier cristal est le plus souvent favorisée par la présence, dans le liquide, de particules étrangères au système et constituées par des impuretés solubles ou insolubles. La germination est alors hétérogène par opposition à la germination homogène qui se produirait dans un liquide idéalement pur, débarrassé de toute surface solide étrangère promotrice de la germination.

La teneur en impuretés insolubles de l'échantillon étudié conditionnant fortement la nature de la phase solide qui apparaît, nous montrerons sous quelle forme cristallise le gallium liquide maintenu en surfusion. Les variétés cristallines métastables seront identifiées aux formes stables à haute pression et à celles qui proviennent de la condensation de la vapeur de gallium. On trouvera par ailleurs [1] des détails sur la partie expérimentale de ce travail.

Germination à partir de la phase liquide. Lorsqu'un cristal, contenant $n$ particules de volume $\boldsymbol{v}$, apparaît dans un liquide, la variation d'enthalpie libre $\Delta G$ du système est donnée [2] par la relation

$$
\Delta G=-n \Delta \mu_{\Delta}+{ }^{2 / 3} a \sigma v^{2 / 3}
$$

dans laquelle $n \Delta \mu=n\left(\mu_{1}-\mu_{\theta}\right)$ représente la variation du potentiel chimique, $\mu_{1}$ et $\mu_{\mathrm{s}}$ sont respectivement les potentiels chimiques d'un atome dans les phases liquide et solide, $a$ est un facteur de forme qui dépend de la géométrie du germe qui apparaît et $\sigma$ est l'enthalpie libre interfaciale rapportée à l'unité de surface entre le solide et le liquide.

Lorsque $n$ croît, la fonction $\Delta G=f(n)$ passe par un maximum $\Delta G^{*}$ qui définit l'enthalpie libre critique.

- Si $n<n^{*}$, la théorie des fluctuations [3] prévoit que de petits agrégats d'atomes se forment par hasard, mais ils disparaissent puisque leur croissance correspondrait à une augmentation de l'en. thalpie libre.

Par contre, si $n>n^{*}$, les agrégats correspondants, appelés germes supercritiques, pourront croître. Cependant, la croissance d'un agrégat suppose un transfert des atomes du liquide à la surface de cet agrégat et nécessite une certaine énergie d'activation $\Delta U$ qui dépend de la diffusion ou de la viscosité de la phase liquide. On sait [3] que la vitesse de germination $J$, c'est-à-dire le nombre de germes 
apparaissant par unité de temps et par unité de volume à la température $T$, est donnée par la relation :

$$
J=K \exp [-\Delta L / k T] \exp \left[-\Delta G^{*} / k T\right]
$$

$K$ est un coefficient cinétique qui dépend de la substance et $k$ est la constante de Boltzmann. Pour les métaux, la mobilité des atomes est grande et le terme exp $[-\Delta U / k T]$ voisin de 1 , si bien que la vitesse de germination est donnée par :

$$
J \simeq K \exp \left[-\Delta G^{*} / k T\right]
$$

Nous nous limiterons au cas des métaux pour lesquels la vitesse de cristallisation est très grande aux températures considérées [4]. L'étude de la variation de $J$ en fonction de la température montre qu'au-dessous du point de fusion $T_{\mathrm{f}}$ et jusqu'à une certaine température $T_{0}$, la vitesse de germination est pratiquement nulle et le métal peut être conservé en surfusion pendant un temps illimité. L'intervalle de température compris entre $T_{\mathrm{f}}$ et $T_{0}$ définit le domaine de métastabilité d'Ostwald.

En explicitant $\Delta G^{*}[2]$

$$
\Delta G^{*}=k B \sigma^{3} /\left(T_{\mathrm{f}}-T\right)^{2}
$$

la relation (2) s'écrit, en première approximation :

$$
J \simeq K \exp \left[-B \sigma^{3} / T\left(T_{\mathrm{f}}-T\right)^{2}\right]
$$

où $B$ est une constante caractéristique de la substance, son expression étant :

$$
B=(4 / 27) a^{3} v^{2} T_{\mathrm{f}}^{2} / k L_{\mathrm{f}}^{2}
$$

dans laquelle $L_{\mathrm{f}}$ désigne la chaleur latente de fusion.

Lorsque la germination est hétérogène, le travail de formation du germe est diminué par la présence des impuretés insolubles "actives ", car la vitesse de germination $J_{\mathrm{B}}[2]$ est alors égale à :

$$
J_{\mathrm{s}}=K \exp \left[-\left(B \sigma^{3} / T\left(T_{\mathrm{f}}-T\right)^{2}\right) g(\theta)\right]
$$

où $g(\theta)=(1-\cos \theta)^{2}(2+\cos \theta) / 4$ est une fonction de l'angle de raccordement $\theta$ entre le germe et l'impureté au sein du liquide.

Surfusion et germination du gallium. - Le gallium, dont la forme stable à la pression atmosphérique $\mathrm{Ga}_{\alpha}$ (ou $\mathrm{Ga} I$ ) fond à $T_{\alpha}=29,78{ }^{\circ} \mathrm{C}$, reste facilement en surfusion, probablement [5] à cause de sa structure cristalline assez particulière : ainsi, lorsqu'une masse liquide de quelques grammes a été chauffée vers $100^{\circ} \mathrm{C}$ dans une solution diluée d'acide chlorhydrique, la solidification ne se produit que vers - $40^{\circ} \mathrm{C}$. Mais il existe une relation entre la température $T^{+}$à laquelle le liquide a été chauffé au-dessus du point de fusion et la température $T$ - à laquelle cesse la surfusion [6].

Cette relation entre $T^{+}$et $T-$ s'explique par la présence, dans le système, d'impuretés que Danilov [I] appelle impuretés " artificiellement actives ", c'est-à-dire d'impuretés qui ne deviennent promotrices de la germination que si elles ont été préalablement en contact avec la substance solide. Au contraire, une impureté " naturellement active " est une impureté qui catalyse la germination sans activation préalable. On a montré que, dans le cas du gallium [8] comme dans celui du bismuth [9] les impuretés naturellement et artificiellement actives sont des produits d'oxydation de ces propres métaux.

En étudiant la surfusion de masses de plusieurs grammes de gallium, nous avons signalé [10] que le liquide pouvait cristalliser en une forme solide métastable fondant à $T_{\beta}=-16,3^{\circ} \mathrm{C}$ : le gallium $\beta$. Avec des masses de plusieurs grammes l'apparition de cette phase est un phénomène très aléatoire à moins que le liquide ne contienne des traces de plomb ou d'étain [11]. Nous avons étudié ensuite la surfusion d'échantillons de quelques microgrammes environ, dans le but d'obtenir des gouttelettes exemptes d'impuretés insolubles. En effet, considérons une masse liquide de plusieurs grammes contenant $m_{0}$ impuretés insolubles favorisant la solidification de la substance. Si l'on divise cette masse en $m$ gouttelettes isolées les unes des autres et si $m \gg m_{0}$, la probabilité pour une gouttelette d'être exempte d'impuretés insolubles est très grande, et par conséquent celle de réaliser les conditions de la germination homogène l'est aussi. Pratiquement cette dispersion s'obtient en agitant la masse métallique liquide dans une phase continue contenant un agent émulsifiant ; dans la plupart de nos expériences la phase continue est l'alcool éthylique et l'agent émulsifiant l'oléate de sodium; les gouttelettes que nous choisissons ont des diamètres qui s'échelonnent entre 10 et $800 \mu \mathrm{m}$.

Pour déterminer les changements de phase dans ces échantillons, nous avons préféré [1] aux méthodes dilatométrique [13] ou optique [12] une méthode de microanalyse thermique différentielle dont la description a été donnée par ailleurs [14]. Dans certains cas, la tension prélevée aux bornes des thermocouples montés en opposition a été amplifiée par un amplificateur à courant continu ayant un gain de $10^{4}$. Ce dispositif [1] permet alors de déceler des quantités de chaleur de l'ordre de $2 \times 10^{-8}$ calories correspondant à la chaleur mise en jeu à la solidification d'une gouttelette de gallium de $10 \mu \mathrm{m}$ de diamètre. Une gouttelette liquide provenant de l'émulsion est donc disposée sur l'une des soudures du couple thermoélectrique et l'ensemble est refroidi.

En général, la solidification se produit, suivant les échantillons, entre -60 et $-123{ }^{\circ} \mathrm{C}$ [1]. Outre le fait que le domaine de température dans lequel le métal liquide reste en surfusion est grandement augmenté - plus de $150^{\circ} \mathrm{C}$ en dessous du point de fusion $T_{\alpha}$ du gallium $\alpha$ - le résultat le plus caractéristique est que la phase solide qui apparait 
dans la presque totalité des cas est la forme métastable Gaß.

Si l'échantillon a un volume $V$ et si $t$ est le temps nécessaire pour que cesse la surfusion à une température $T$, la fréquence de germination $I$, c'est-àdire le nombre de germes apparaissant par unité de temps, est proportionnelle à :

$$
\begin{aligned}
\frac{1}{t}=J V=K V & \exp \left[-\frac{\Delta G^{*}}{k T}\right] \\
& =K V \exp \left[-\frac{B \sigma^{3}}{T\left(T_{\mathrm{f}}-T\right)^{2}}\right]
\end{aligned}
$$

Si $I$ est constant, on voit qu'une relation appelée " isocinétique " [15] doit exister entre le volume $V$ et la température $T$. Cependant, cette interprétation suppose qu'à tout instant, une population d'agrégats existe dans la phase liquide ; Buckle [16] a montré qu'au début de la transformation, il se passe un temps de relaxation $\tau$ pendant lequel la concentration d'équilibre s'établit: cet auteur admet que $\tau$ est voisin du temps expérimental $t$ et est défini par le rapport du nombre d'atomes $n_{1}$ par unité de volume du liquide à la vitesse de germination, $\tau=n_{\mathrm{l}} / J$ et il en déduit que la température de solidification est pratiquement indépendante du volume.

Si l'on divise en $m$ gouttes de même volume une masse initiale contenant $m_{0}$ particules en suspension, il apparaît que :

- Si $m \gg m_{0}$, la germination doit être homogène dans la plupart des gouttes choisies, la courbe donnant la température $T$ de solidification spontanée sera représentée par $a b$ (fig. 1a).

- Si $m \ll m_{0}$, les gouttes contiennent au moins une impureté et la germination est hétérogène (courbe $c e$ ).

- Enfin, si $m \simeq m_{0}$, les échantillons cristallisent à n'importe quelle température comprise entre les températures définies par $b$ et $c$. Cette interprétation suppose que les impuretés non solubles sont de même nature et elle ne tient pas compte de la concentration dans chaque goutte ; en réalité, il n'y aura pas de discontinuité mais une courbe dont l'allure est représentée par $b^{\prime} c^{\prime}[15]$.

Sur la figure $1 b$ sont portées les températures minimales de germination spontanée en fonction du diamètre des gouttelettes de gallium, ces diamètres étant compris entre $10 \mu \mathrm{m}$ et $1 \mathrm{~mm}$. Sur la portion $a^{\prime} b^{\prime}$ de la courbe, la température de cristallisation d'un même échantillon est reproductible à $\pm 1^{\circ} \mathrm{C}$ alors que pour des échantillons de diamètres supérieurs à $80 \mu \mathrm{m}$ la dispersion des températures peut atteindre $30{ }^{\circ} \mathrm{C}$. Des résultats absolument identiques ont été obtenus avec des émulsions où les échantillons étaient entourés d'un film de nature différente [1] et nous pensons que les conditions de la germination homogène ont été réalisées pour les
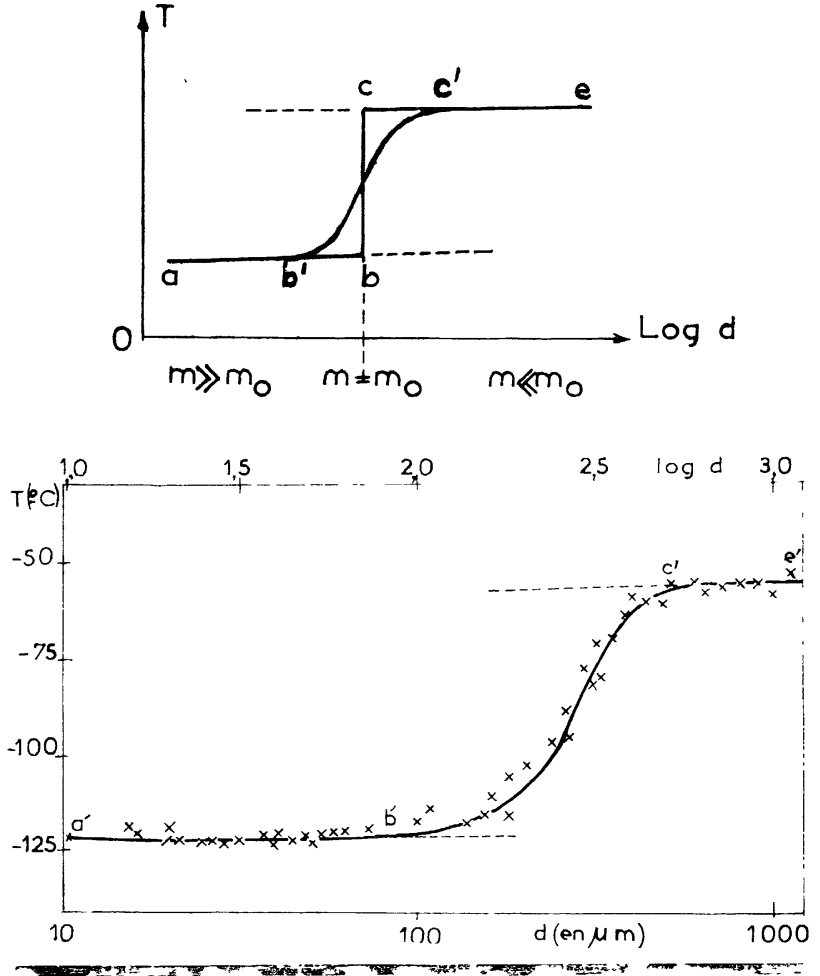

FIg. 1. - a) Influence du diamètre des gouttelettes sur la température à laquelle cesse la surfusion (d'après [15]).

b) Courbe expérimentale relative au gallium.

gouttelettes de diamètre inférieur à $80 \mu \mathrm{m}$; au delà de $80 \mu \mathrm{m}$ la germination devient hétérogène.

Jusqu'ici nous n'avons considéré que la température à laquelle cesse la surfusion sans tenir compte de la forme solide qui apparaît ; précisons cependant qu'aux températures qui correspondent à la portion $a^{\prime} b^{\prime}$ de la courbe, nous avons toujours obtenu la forme métastable $\mathrm{Ga} \beta$ qui fond à $-16,3^{\circ} \mathrm{C}$.

L'enthalpie libre interfaciale. - L'expression (7) de la fréquence de germination relative à $\mathrm{Ga} \beta$

$$
I=1 / t=K V \exp \left[-B \sigma_{\beta}^{3} / T\left(T_{\beta}-T\right)^{2}\right]
$$

montre que, pour un échantillon donné, il existe une relation entre le temps $t$ nécessaire à la germination de l'échantillon de volume $V$ et la température à laquelle la solidification se produit. On a donc un moyen de déterminer l'énergie libre inter. faciale rapportée à l'unité de surface entre un germe de $\mathrm{Ga}_{\beta}$ et le liquide. Dans cette expression, on ne tient pas compte de l'influence de la courbure du germe sur la valeur de l'enthalpie libre interfaciale ; on peut rappeler les études théoriques de Tolman [17] et Kirkwood et Buff [18] qui proposent la relation : $\sigma=\sigma_{0} /[1+2 \delta / r]$ entre les enthalpies libres superficielles $\sigma_{0}$ pour une surface plane $(r=\infty)$ et $\sigma$ 
pour un germe de rayon $r, \delta$ étant voisin de $10^{-8} \mathrm{~cm}$.

Un échantillon de diamètre inférieur à $80 \mu \mathrm{m}$ et cristallisant spontanément vers $-120{ }^{\circ} \mathrm{C}$ est disposé sur la soudure d'un dispositif de mesure thermique différentielle, puis immergé dans un bain maintenu à différentes températures $T$ constantes à $\pm 0,02{ }^{\circ} \mathrm{C}$. On mesure le temps nécessaire à la cristallisation de l'échantillon [1].

Sur la figure 2 nous montrons, à titre d'exemple, la variation de $\log 1 / t$ ( $t$ est exprimé en secondes) en fonction $1 / T\left(T_{\beta}-T\right)^{2}$ pour un échantillon de diamètre égal à $28,5 \mu \mathrm{m}$.

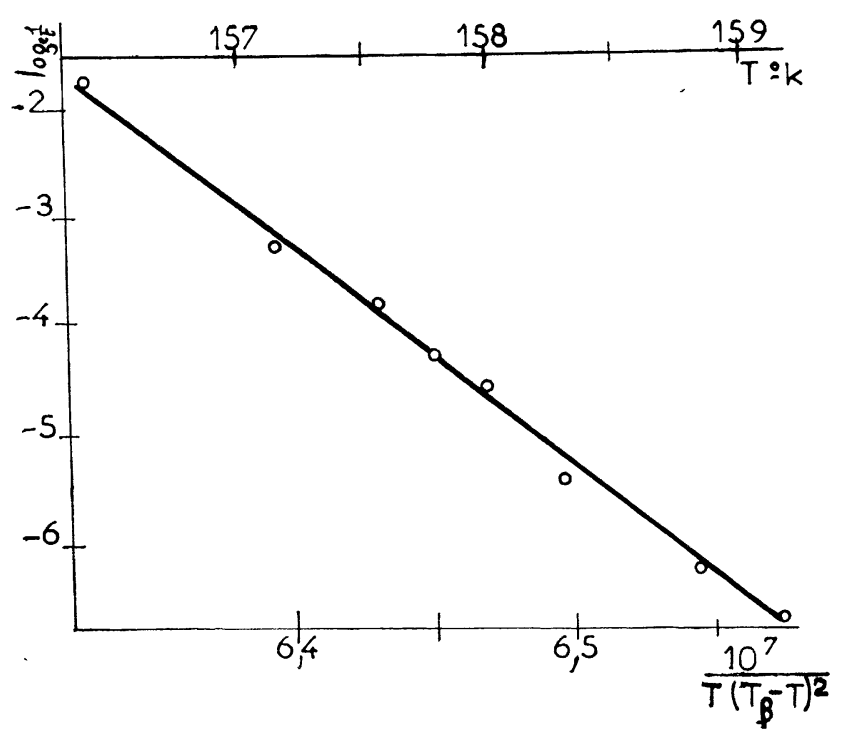

Fig. 2. - Variation de $\log 1 / t$ ( $t$ étant exprimé en secondes) en fonction de $10^{7} / T\left(T_{\beta}-T\right)^{2}$.

Pour différents échantillons de diamètres compris entre 19 et $53 \mu \mathrm{m}$, nous avons trouvé :

$$
\sigma_{\beta}=38,1 \pm 0,8 \mathrm{ergs} / \mathrm{cm}^{2} \text {. }
$$

Turnbull a établi [19] que l'enthalpie libre interfaciale d'une substance est sensiblement proportionnelle à $L_{\mathrm{f}}$ et $\rho^{2 / 3}$ si $L_{\mathrm{f}}$ et $\rho$ sont respectivement la chaleur latente de fusion et la masse volumique du solide. A partir de $\sigma_{\beta}=38,1 \mathrm{ergs} / \mathrm{cm}^{2}$, on peut donc déterminer un ordre de grandeur de l'énergie interfaciale $\sigma_{\alpha}$ entre un germe de la forme stable $\alpha$ et le liquide. On trouve $\sigma_{\alpha} \simeq 77 \mathrm{ergs} / \mathrm{cm}^{2}$.

La relation (3) permet de calculer le rapport $\Delta G_{\beta}^{*} / \Delta G_{\alpha}^{*}=0,7$ de l'enthalpie libre critique des germes de $\mathrm{Ga}_{\beta}$ à celle des germes de $\mathrm{Ga}_{\alpha}$ vers $158^{\circ} \mathrm{K}$. Comme $\Delta G_{\beta}<\Delta G_{\alpha}$, il en résulte que la fréquence de germination $I_{\beta}$ est supérieure à $I_{\alpha}$.

- Dans le domaine de température où la germination est homogène, la cristallisation du liquide en la phase $\mathrm{Ga}_{\alpha}$ sera donc exceptionnelle, la probabilité pour qu'un germe de gallium $\beta$ apparaisse étant beaucoup plus grande. Ce fait est confirmé expéri- mentalement : sur 1500 essais effectués sur des échantillons différents, la forme $\alpha$, c'est-à-dire la forme normale stable, n'est jamais apparue.

La forme cristalline Ga . - MISE EN ÉvIDENCE. - En opérant [20] sur certains échantillons de diamètre inférieur à $800 \mu \mathrm{m}$, nous avons parfois observé les phénomènes thermiques suivants (fig. 3).

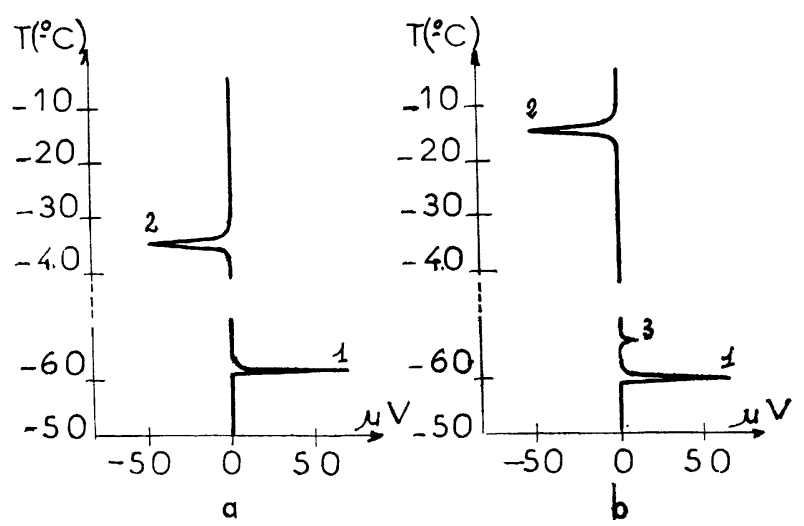

Fig. 5. - Thermogrammes obtenus par A. T. D. avec certaines gouttes de gallium :

a) Signal 1: cristallisation en Gar; signal 2 : fusion de Gar.

b) Signal 1 : cristallisation en Gar ; signal 3 : transformation en $\mathrm{Ga} \beta$; signal 2 : fusion de Gaß.

A une température comprise entre - 60 et $-105^{\circ} \mathrm{C}$, il se produit un dégagement de chaleur (signal 1, fig. $3 a$ ) qui correspond à la solidification du liquide ; or, par réchauffement, on remarque un pic endothermique (signal 2) à $-35,6{ }^{\circ} \mathrm{C}$, aucun autre phénomène n'est observé aux températures supérieures, en particulier à $-16,3^{\circ} \mathrm{C}$ (point de fusion du gallium $\beta$ ), ni à $29,78^{\circ} \mathrm{C}$ (point de fusion du gallium $\alpha$ ).

Avec ces mêmes gouttes, nous avons très souvent observé, après un premier pic exothermique (signal 1 fig. $3 b$ ) qui correspond à la solidification du liquide, un deuxième dégagement de chaleur (signal 3) beaucoup plus faible que le précédent ; dans ce cas, en élevant la température nous obtenons un pic endothermique à $-16,3^{\circ} \mathrm{C}$ (signal 2). Cette dernière expérience montre que le gallium s'est solidifié en une forme solide (premier signal exothermique) qui s'est ensuite transformée en $\mathrm{Ga}_{\beta}$ et celle-ci fond à $-16,3^{\circ} \mathrm{C}$. Les mêmes phénomènes thermiques ont été retrouvés avec des échantillons de gallium de différentes origines et sur des émulsions où les gouttelettes étaient enrobées par des films de natures différentes [21].

Une deuxième forme métastable de gallium fondant à $-35,6^{\circ} \mathrm{C}$ a donc été mise en évidence ; nous l'appellerons gallium $\gamma$. La mesure de quelques propriétés physiques et l'étude aux rayons $\mathrm{X}$ confirment l'existence de cette nouvelle forme. 
Chaleur latente de fusion. - Pour mesurer la chaleur latente de fusion, nous avons utilisé un dispositif d'analyse thermique différentielle [22] assez comparable à celui mis en œuvre précédemment. Cependant l'une des soudures des couples montés en opposition a été creusée d'une cavité dans laquelle la goutte de gallium est disposée. La chaleur latente de fusion de l'autre forme solide $\mathrm{Ga}_{\beta}$ connue et égale à $9,1 \mathrm{cal} / \mathrm{g}$ [23] a permis d'étalonner le calorimètre.

$\mathrm{A}-35,6^{\circ} \mathrm{C}$, la chaleur latente de fusion de la forme $\gamma \mathrm{du}$ gallium ainsi mesurée est de $8,35 \pm 0,15 \mathrm{cal} / \mathrm{g}$.

Les variations d'entropie à la fusion des phases métastables $\gamma$ et $\beta: \Delta S_{\gamma}=2,45 \mathrm{cal}$. mole ${ }^{-1}$. deg-1 et $\Delta S_{\beta}=2,47$ cal.mole-1. $\mathrm{deg}^{-1}$, sont beaucoup plus proches de celles des métaux à structure compacte (voisines de $2,2 \mathrm{cal}^{-m_{0} e^{-1}} \cdot \mathrm{deg}^{-1}$ ) que la variation d'entropie à la fusion de la forme solide stable $\Delta S_{\alpha}=4,41$ cal. mole $-1 . \operatorname{deg}^{-1}[24]$.

Variation de volume. - Les variations de volume accompagnant la fusion des échantillons ont été mesurées à l'aide d'un microdilatomètre à tige [25]. Sur la figure 4 on montre, par exemple,

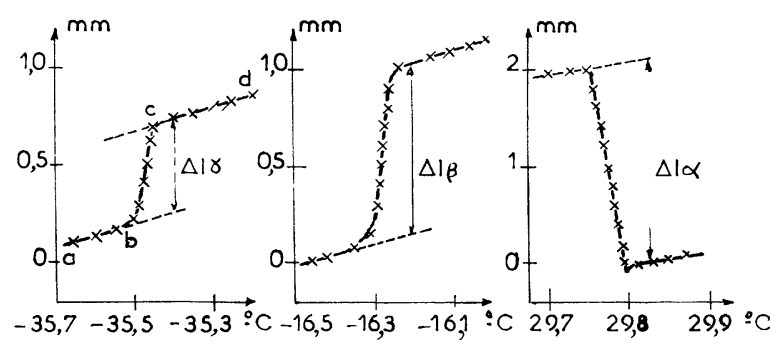

Fig. 4. - Variations du niveau dilatométrique accompagnant respectivement les fusion des formes $\alpha, \beta, \gamma$.

pour un échantillon de $700 \mu \mathrm{m}$ de diamètre, la variation du niveau dilatométrique en fonction de la température, celle-ci variant de $2{ }^{\circ} \mathrm{C} / \mathrm{h}$. Les écarts $\Delta \mathrm{l}_{\gamma}, \Delta \mathrm{l}_{\beta}, \Delta \mathrm{l}_{\alpha}$ donnent respectivement la dénivellation à la fusion de chacune des trois formes allotropiques du gallium pour la même gouttelette.

La variation de volume accompagnant la fusion de la phase stable $\alpha$ étant égale à $\Delta V_{\alpha}=0,00529$ $\mathrm{cm}^{3} / \mathrm{g}$, on peut déduire de ces mesures :

$$
\begin{aligned}
& \Delta V_{\gamma}=0,00136 \pm 0,00008 \mathrm{~cm}^{3} / \mathrm{g} \\
& \Delta V_{\boldsymbol{\beta}}=0,0023 \pm 0,0001 \mathrm{~cm}^{3} / \mathrm{g} .
\end{aligned}
$$

En utilisant une émulsion de métal liquide, nous avons déterminé la masse volumique du liquide à $-35,6^{\circ} \mathrm{C}$ et à $-16,3^{\circ} \mathrm{C}$ :

$$
\begin{aligned}
& \rho_{1}\left(-35,6^{\circ} \mathrm{C}\right)=6,153 \pm 0,008 \mathrm{~g} / \mathrm{cm}^{3} \\
& \rho_{1}\left(-16,3^{\circ} \mathrm{C}\right)=6,136 \pm 0,005 \mathrm{~g} / \mathrm{cm}^{3} .
\end{aligned}
$$

Par suite les masses volumiques de Ga $\gamma$ et $\mathrm{Ga}_{\beta}$ sont respectivement :

$$
\rho_{\gamma}=6,20 \pm 0,01 \mathrm{~g} / \mathrm{cm}^{3} \text { à }-35,6^{\circ} \mathrm{C}
$$

et

$$
\rho_{\beta}=6,23 \pm 0,01 \mathrm{~g} / \mathrm{cm}^{3} \text { à }-16,3{ }^{\circ} \mathrm{C} .
$$

On remarquera que la forme métastable $\mathrm{Ga}_{\gamma}$ est moins dense que l'autre phase instable $\mathrm{Ga}_{\beta}$.

Étude cristallographique. - L'étude cristallographique de Gar [26] a été faite en collaboration avec le Laboratoire de Minéralogie Cristallographie de la Sorbonne par une méthode analogue à celle déjà utilisée pour déterminer la structure du gallium $\beta$.

Quatre séries de clichés ont permis d'obtenir des représentations cotées du réseau dans des orientations différentes.

La maille est orthorhombique de type $\mathrm{C}$, avec les paramètres suivants : $a=10,60 \pm 0,07 \AA$, $b=13,56 \pm 0,04 \AA$ et $c=5,19 \pm 0,04 \AA$. En utilisant les résultats rëlatifs à la masse volumique : $p_{\gamma}=6,20 \mathrm{~g} / \mathrm{cm}^{3}$ et à la valeur moyenne $\mathrm{du}$ volume de la maille élémentaire nous trouvons $Z=40$ atomes par maille.

L'indexation des taches montre que les seuls groupes de symétrie à retenir sont :

$$
C_{2 \mathrm{v}}^{12}\left(\mathrm{Cm} \mathrm{c} 2_{1}\right), \quad C_{2 \mathrm{v}}^{16}(\mathrm{C} 2 \mathrm{~cm}) \text { et } D_{2 \mathrm{~h}}^{17}(\mathrm{Cm} \mathrm{cm}) .
$$

Sur le tableau 1 sont réunies les principales propriétés cristallographiques et thermodynamiques du gallium dans les états liquide et cristallin, à la pression atmosphérique.

Le diagramme température-pression du gallium. - Nous essaierons d'établir une relation entre les diagrammes d'équilibre à haute pression et les données thermodynamiques à la pression atmosphérique ; la forme métastable Gar peut en effet posséder un domaine de stabilité aux pressions élevées puisque sa masse volumique est plus grande que celle de la forme stable Ga $\alpha$.

La difficulté d'établir à partir des données thermodynamiques le domaine pression-température dans lequel une substance peut exister sous une forme cristalline donnée provient des faibles différences énergétiques entre les diverses modifications solides ; par exemple, la différence entre les enthalpies libres des formes cubique diamant et quadratique de l'étain est seulement de $0,022 \mathrm{eV} /$ atome (valeur rapportée à $\left.0{ }^{\circ} \mathrm{K}\right)$ [24]. Les différences énergétiques entre les modifications cristallines d'une même substance étant aussi faibles, il n'est pas étonnant que l'on puisse obtenir parfois des formes, à l'état métastable, dans le domaine température-pression de stabilité d'une autre phase solide. A titre d'exemple considérons le cas de la glace dont plusieurs formes cristallines (les glaces II, III, V, V I et VII) ont leur 
TABLEAU I

\begin{tabular}{|c|c|c|}
\hline Point de fusion $\left({ }^{\circ} \mathrm{C}\right)$ & Ga LIQUIDE & $\begin{array}{l}\mathrm{Ga}_{\boldsymbol{\gamma}} \\
-\overline{35,6}\end{array}$ \\
\hline Système cristallin ... & & orthorhomb. \\
\hline \multicolumn{3}{|l|}{ Groupe spatial ..... } \\
\hline $\begin{array}{l}\text { Paramètres de la } \\
\text { maille }(\text { en } \AA) \ldots \ldots\end{array}$ & & $\begin{array}{l}a=10,60 \pm 0,07 \\
b=13,56 \pm 0,04 \\
c=5,19 \pm 0,04\end{array}$ \\
\hline $\begin{array}{c}\text { Nombre d'atomes par } \\
\text { maille } \ldots \ldots \ldots \ldots . .\end{array}$ & & 40 \\
\hline $\begin{array}{r}\text { Distances d'un atome } \\
\text { à ses voisins (en } \AA \text { ) }\end{array}$ & $\begin{array}{l}2,83[34] \\
2,77[35]\end{array}$ & \\
\hline $\begin{array}{c}\text { Remarques } \quad \text { sur la } \\
\text { structure } \ldots \ldots \ldots\end{array}$ & & \\
\hline $\begin{array}{c}\text { Masse volumique (en } \\
\left.\mathrm{g} / \mathrm{cm}^{3}\right) \ldots \ldots \ldots \ldots\end{array}$ & $\begin{aligned} & 6,095 \\
\left(\text { à } 29,8{ }^{\circ} \mathrm{C}\right. & \end{aligned}$ & $\begin{array}{c}6,20 \pm 0,01 \\
\left(\grave{\mathrm{a}}-\overline{35}, 6^{\circ} \mathrm{C}\right)\end{array}$ \\
\hline $\begin{array}{l}\text { Chaleur latente de fu- } \\
\text { sion (en cal/g) .... }\end{array}$ & & $8,35 \pm 0,15$ \\
\hline $\begin{array}{l}\text { Résistivité élec. (en } \\
10^{-6} \Omega . \mathrm{cm} \quad \dot{a} \\
\left.-20^{\circ} \mathrm{C}\right) \ldots \ldots \ldots\end{array}$ & 24,86 & \\
\hline
\end{tabular}

domaine de stabilité aux pressions élevées. Des expériences récentes ont montré que vers $-180^{\circ} \mathrm{C}$, ces formes obtenues à haute pression, peuvent être conservées à la pression atmosphérique [36]. L'existence de phases métastables dans le domaine est stabilité d'une autre forme solide est également bien vérifiée dans le cas du gallium $I I$. Sur la figure 5 nous rappelons le diagramme d'état du gallium, établi par Bridgman [37] en 1935 : les coordonnées du point triple $L-I-I I$ sont $2,4^{\circ} \mathrm{C}$ et $11,9 \mathrm{kbars}$,

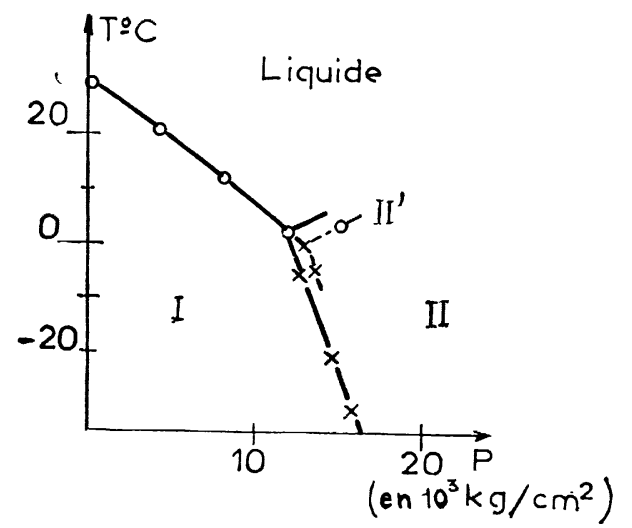

Fig. 5. - Diagramme d'état du gallium, établi par Bridgman (1935).

\begin{tabular}{|c|c|}
\hline $\mathrm{Ga}_{\beta}$ & $\mathrm{Ga}_{\alpha}$ \\
\hline$-16,3$ & $+29,78$ \\
\hline orthorhomb. & orthorhomb. [28] \\
\hline $\mathrm{D}_{2 \mathrm{~h}}^{17}[27]$ & $\mathrm{D}_{2 \mathrm{~h}}^{18}[28]$ \\
\hline $\begin{array}{l}2,90 \pm 0,03 \\
8,13 \pm 0,03 \\
3,17 \pm 0,03[27]\end{array}$ & $\begin{array}{l}a=4,5259 \\
b=4,5199 \\
c=7,6603[29]\end{array}$ \\
\hline $4[27]$ & $8[28]$ \\
\hline
\end{tabular}

$$
\begin{array}{lcl}
2 & \text { voisins à } & 2,68 \\
4 & \cdots & 2,85 \\
2 & » & 2,90 \\
2 & \cdots & 3,17[27]
\end{array}
$$

Les atomes sont groupés en chaines en zig-zag [27]

$$
\begin{gathered}
6,23 \pm 0,01 \\
\left(\grave{\mathrm{a}}-16,3^{\circ} \mathrm{C}\right) \\
9,09 \pm 0,03
\end{gathered}
$$

$8,3<\rho \mathrm{Ga}_{\beta}<11,3$ Anisotropie très réduite [33]
1 voisin à 2,484

$2 \quad 2,691$

2 ॥ 2,730

2 » $2,788[29]$

Chaque atome a un seul voisin très rapproché [28].

$$
\begin{gathered}
5,904[30] \\
\text { à } 29,78^{\circ} \mathrm{C} \\
19,14 \pm 0,01[31]
\end{gathered}
$$

$6,9<\rho \mathrm{Ga}_{\alpha}<46,5[32]$ la forme Ga $I I^{\prime}$ apparaît " capricieusement " à l'état métastable, dans le domaine de stabilité de Ga $I I$; on sait [6] que la forme Gaß s'identifie à la forme Ga $I I$ qui apparaît à la pression atmosphérique. Nous avions suggéré [20] que la forme métastable Gar pouvait correspondre à une forme solide stable à haute pression mais les données concernant le diagramme d'état aux pressions élevées ne nous permettaient pas alors de vérifier cette hypothèse. Or, récemment Jayaraman et al. [38] ont fait une nouvelle étude du polymorphisme du gallium à haute pression et mis en évidence une troisième forme solide $\mathrm{Ga} I I I$ (fig. 6) le point triple $\mathrm{Ga} I I$ - Ga $I I I$ - liquide se situant à $45^{\circ} \mathrm{C}$ et 30 kbars; ces auteurs montrent que la variété Ga $I I I$ peut être facilement obtenue à l'état métastable dans le domaine de stabilité de Ga $I I$ et l'identifient à la forme Ga $I I^{\prime}$ signalée par Bridgman. Remarquons que Ga $I I I$ est moins dense que Ga $I I$ et rappelons que $\mathrm{Ga}_{\gamma}$ est également moins dense que $\mathrm{Ga}_{\beta}($ ou $\mathrm{Ga} I I)$; il est alors tentant d'identifier $\mathrm{Ga}_{\gamma}$ à Ga $I I I$.

\section{Si, dans l'équation de Clapeyron-Clausius}

$$
T \mathrm{~d} P / \mathrm{d} T=L_{\mathrm{f}} / \Delta V
$$

appliquée à la fusion d'une substance, on admet [39] 


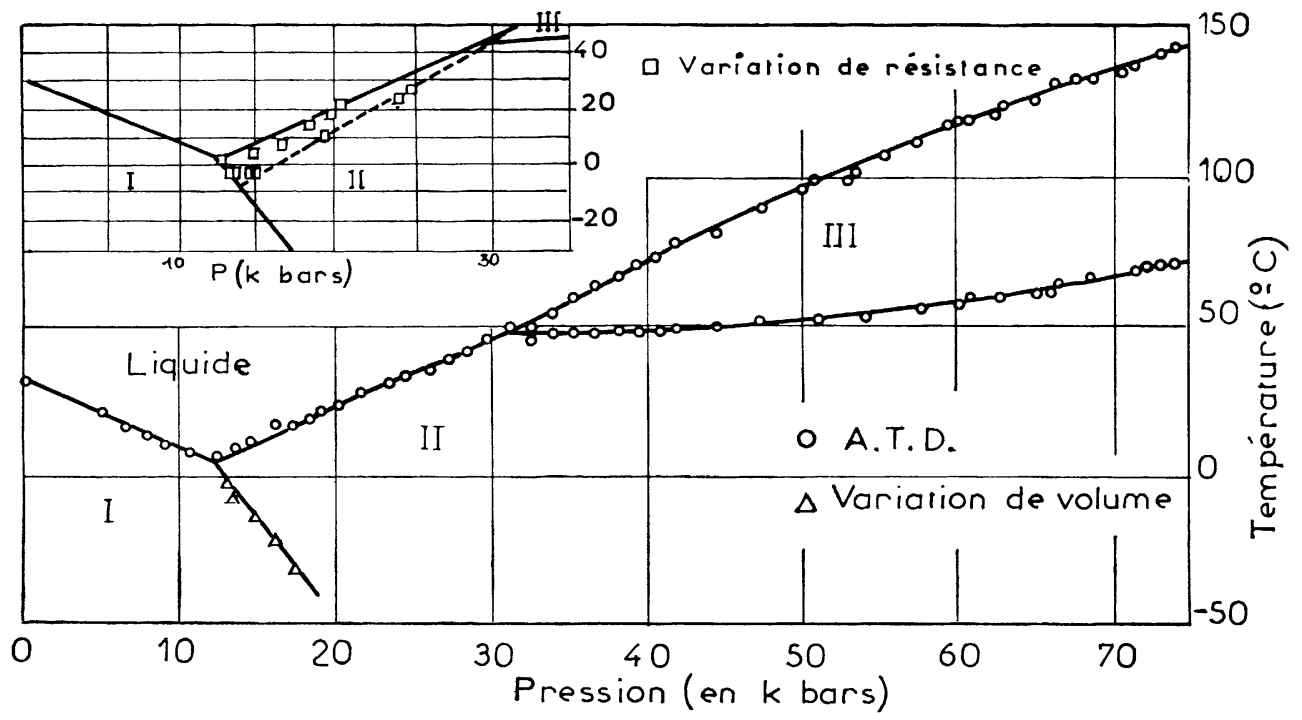

FIg. 6. - Diagramme d'état du gallium publié par Jayaraman et al. (1963).

Dans la partie située en haut et à gauche, les lignes en traits continus délimitent les domaines de stabilité des phases; la ligne en traits discontinus correspond à l'équilibre métastable entre Ga $I I I$ et le liquide.

que le rapport $L_{\mathrm{f}} / \Delta V$ de la chaleur latente de fusion $L_{\mathrm{f}}$ à la variation de volume $\Delta V$ est une fonction linéaire de la pression, on peut écrire :

$$
T \mathrm{~d} P / \mathrm{d} T=L_{\mathrm{f}} / \Delta V=c(P+b)
$$

où $b$ et $c$ sont des constantes.

L'intégration de l'équation (8) conduit à la relation :

$$
\frac{1}{c} \log \frac{P+b}{P_{0}+b}=\log \frac{T}{T_{0}}
$$

$T_{0}$ étant la température de fusion de la substance considérée à une pression $P_{0}$ donnée.

En posant : $a=P_{0}+b$, on obtient la formule de Simon-Glatzel $[40]$

$$
\left(P-P_{0}\right) / a=\left(T / T_{0}\right)^{c}-1
$$

Gilvary [41] a montré que pour les métaux faiblement compressibles, $c$ peut s'exprimer en fonction de la constante de Gruneisen $\gamma$ par la relation $c=\frac{6 \gamma+1}{6 \gamma-2}$ alors que $a$ est étroitement lié à la pression interne du solide.

En réalité $c$ varie avec la pression, le rapport $L_{\mathrm{f}} / \Delta V$ n'étant fonction linéaire de la pression qu'en première approximation : la relation (9) n'est qu'une formule "d'interpolation ». Le tableau II qui donne $L_{\mathrm{f}} / \Delta V$ à la fusion de la modification II du gallium, pour différentes valeurs de la pression, montre que la formule de Simon ne peut être appliquée dans tout l'intervalle s'étendant de la pression atmosphérique à 30 kbars.

Nous avons alors [1] établi une expression analy-
TABLEAU II

$\begin{array}{cc}P \text { en kbars } & L_{\mathrm{f}} \mid \Delta V \times \frac{10^{-3} \mathrm{cal} / \mathrm{cm}^{3}}{-} \\ \text { Pression } & 3,96 \\ \text { atmosphérique } & \\ 11,7 & 2,94 \\ 30 & 3,39\end{array}$

tique de la courbe de fusion de Ga $I I$ à partir des résultats expérimentaux : l'allure de la courbe est bien traduite entre la pression atmosphérique et 30 kbars par une relation de la forme :

$$
T=318\left(\frac{P+4}{3 / 4}\right)^{\mathbf{0 . 2 4 6}}+69 \exp (-0,145 P)
$$

la pression étant exprimée en kilobars et la température en degrés Kelvin; le premier terme correspond à la formule de Simon, le second est un terme correctif.

L'équation de Simon (9) donnant l'expression analytique de la courbe de fusion de la forme solide Ga $I I I$ pour les pressions comprises entre 30 et 70 kbars est :

$$
T=318\left(\frac{P+5}{35}\right)^{\mathbf{0 . 3 1 7}} .
$$

Les propriétés thermodynamiques des deux modifications à haute pression sont très voisines et nous porterons dans l'équation exprimant la courbe de fusion de Ga $I I I$ le même terme correctif $69 \times \mathrm{e}^{-\mathbf{0 . 1 4 5 P}}$; l'équation de la courbe de fusion de cette forme solide est alors, pour les pressions 
comprises entre la pression atmosphérique et 70 kbars :

$$
T=318\left(\frac{P+5}{35}\right)^{\mathbf{0 . 3 1 7}}+69 \mathrm{e}^{-\mathbf{0 . 1 4 5 P}} .
$$

Si l'on admet que Ga $I I I$ peut exister à la pression atmosphérique son point de fusion $T_{I I I}$ et la pente $(\mathrm{d} T / \mathrm{d} P)_{I I I}$ calculés à partir de la relation (10) sont :

$$
T_{I I I}=241{ }^{\circ} \mathrm{K} \text { et }(\mathrm{d} T / \mathrm{d} P)_{I I I}=0,88^{\circ} \mathrm{K} / \mathrm{kbar} \text {. }
$$

Ces valeurs sont très voisines de celles obtenues expérimentalement pour Ga $\gamma$ :

$$
T_{\gamma}=237,5^{\circ} \mathrm{K} \text { et }(\mathrm{d} T / \mathrm{d} P)_{\gamma}=0,92^{\circ} \mathrm{K} / \mathrm{kbar} \text {. }
$$

Enfin Zohkovski [42] a remarqué que pour un grand nombre de substances, la variation de volume $\Delta V$ accompagnant la fusion à la température $T$ ( $T$ étant fonction de la pression) est donnée par la relation empirique :

$$
\Delta V=C \mathrm{e}^{B T}
$$

dans laquelle $C$ et $B$ sont des constantes caractéristiques de la substance. Les variations de volume à la fusion de Ga $I I I$ sont égales à :

$$
\Delta V=0,0035 \mathrm{~cm}^{3} / \mathrm{g} \text { à } 318^{\circ} \mathrm{K}[38]
$$

et

$$
\Delta V=0,0023 \mathrm{~cm}^{3} / \mathrm{g} \text { à } 273{ }^{\circ} \mathrm{K} \text { [37] }
$$

la relation (11) s'applique à Ga $I I I$ et devient

$$
\Delta V=17,5 \times 10^{-5} \mathrm{e}^{9,4 \times 10^{-3} \mathrm{~T}} .
$$

A la pression atmosphérique, pour $T=2 / 1^{\circ} \mathrm{K}$, la variation de volume sera $\Delta V_{I I I}=0,0017 \mathrm{~cm}^{3} / \mathrm{g}$; cette valeur n'est pas très éloignée de $\Delta V_{\gamma}=0,0014$ $\mathrm{cm}^{3} / \mathrm{g}$.

L'identité déjà établie $[6]$ des phases $\mathrm{Ga} \beta$ et $\mathrm{Ga} I I$, les densités de $\mathrm{Ga \gamma}$ et $\mathrm{Ga} I I I$ respectivement plus faibles que celles de $\mathrm{Ga} \beta$ et de Ga $I I$, la solidification occasionnelle de la phase Ga III dans le domaine de stabilité de la forme Ga $I I$, l'accord entre les valeurs de $T_{\gamma}$ et de $T_{I I I}$, de $(\mathrm{d} T / \mathrm{d} P)_{\gamma}$ et de $(\mathrm{d} T / \mathrm{d} P)_{I I I}$, de $\Delta V_{\gamma}$ et de $\Delta V_{I I I}$ nous permettent de supposer que la phase Ga $\gamma$, mise en évidence à la pression atmosphérique à partir du liquide maintenu en surfusion, est la forme Ga $I I I$, stable aux pressions supérieures à 30 kbars.

Conditions d'apparition des formes métas. tables de gallium. - $a$ ) A PARTIR DE LA PHASE IIQUIDE. - Nous appuyant sur une étude concernant un millier de gouttelettes de diamètres compris entre 10 et $800 \mu \mathrm{m}$, nous avons porté sur la figure 7 le pourcentage des échantillons qui, à une température donnée, cristallisent soit en Gaß soit en Ga $\gamma$.

On voit que si la cristallisation se produit entre -55 et $-100^{\circ} \mathrm{C}$, la probabilité d'apparition de

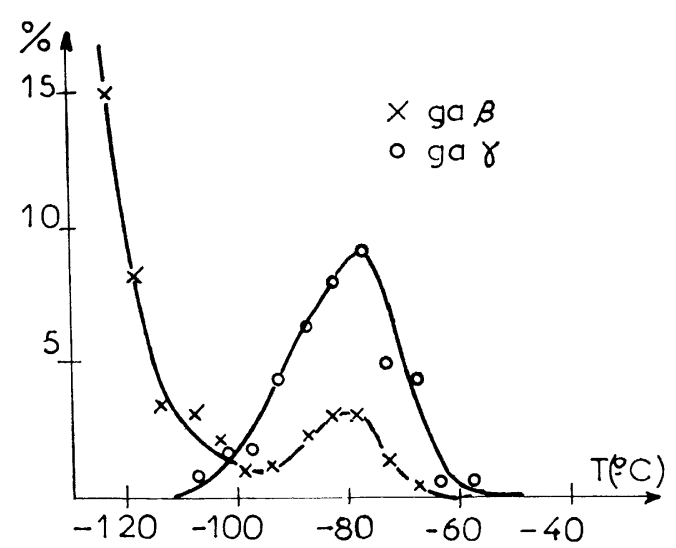

Fig. 7. - Pourcentage des gouttes qui cristallisent à une température donnée en $\mathrm{Ga} \beta$ ou en Ga $\gamma$.

la forme $\gamma$ est plus grande que celle de la forme $\beta$, tandis qu'au-dessous de $-115^{\circ} \mathrm{C}$, on obtient toujours $\beta$.

Un fait intéressant est que la présence d'un produit d'oxydation du gallium semble favoriser la formation de Gar. En effet, soit une gouttelette cristallisant à $-120^{\circ} \mathrm{C}$ en $\beta$; si l'on déchire légèrement le film qui enrobe la goutte, permettant ainsi une faible oxydation du métal liquide, on constate que la surfusion cesse à des températures plus élevées variant entre -70 et $-90^{\circ} \mathrm{C}$; dans ce cas c'est le plus souvent la phase solide Gay qui apparaît. (Notons que si le film adsorbé à la surface de l'échantillon métallique est entièrement détruit, la goutte liquide cristallise entre -10 et $-48{ }^{\circ} \mathrm{C}$ en la forme stable $\mathrm{Ga}_{\alpha}$.)

Il est possible d'expliquer ces résultats en comparant les variations d'enthalpie libre $\Delta G_{\gamma}^{*}$ et $\Delta G_{\beta}^{*}$ accompagnant la cristallisation du liquide respectivement en $\mathrm{Ga}_{\gamma}$ et $\mathrm{Ga}_{\beta}$. En effet, l'enthalpie libre interfaciale $\sigma_{\gamma}$ entre un germe de gallium $\gamma$ et le liquide, calculée [15] à partir de la valeur expérimentale $\sigma_{\beta}$, est $\sigma_{\gamma} \simeq 34 \mathrm{ergs} / \mathrm{cm}^{2}$; si la germination est homogène, à une température voisine de $-115^{\circ} \mathrm{C}, \Delta G_{\gamma}^{*}=1,2 \Delta G_{\beta}^{*}$ et la fréquence de germination (formule 2) de $\mathrm{Ga}_{\beta}$ est supérieure à celle de $\mathrm{Ga}_{\gamma}$.

Si la germination est hétérogène, les impuretés diminuent le travail de formation des germes (formule 6) et le terme $J_{\mathrm{s}}$ correspondant à la vitesse de germination de $\mathrm{Ga}_{\gamma}$ devient supérieur à celui du gallium $\beta$.

b) A partir de la phase vapeur. - Bückel et Gey [43] ont étudié la supraconductivité des films de gallium provenant de la condensation de la vapeur sur des supports de quartz; lorsque le support est maintenu à $4^{\circ} \mathrm{K}$, le dépôt obtenu serait " amorphe " et sa température critique de supraconductibilité est $T_{\mathrm{Cl}}=8,4^{\circ} \mathrm{K}$ alors que la gallium 
stable Ga $\alpha$ possède la température de transition $T_{\mathrm{C} \alpha}=1,07^{\circ} \mathrm{K}$. Par réchauffement, l'état " amorphe» du gallium se transforme vers $20^{\circ} \mathrm{K}$ en une forme cristalline dont la température de transition est $6,5^{\circ} \mathrm{K}$ et identifiée par Bückel au gallium $I I$ (ou $\beta$ ).
Par un réchauffement ultérieur, vers 70 ou 80 oK, il se produit une transformation en gallium $\alpha\left({ }^{1}\right)$.

Sur le tableau III nous indiquons les domaines de température où les formes de gallium peuvent exister à la pression atmosphérique.

\section{TABLEAU III}

A) Condensation de la phase vapeur en couches minres

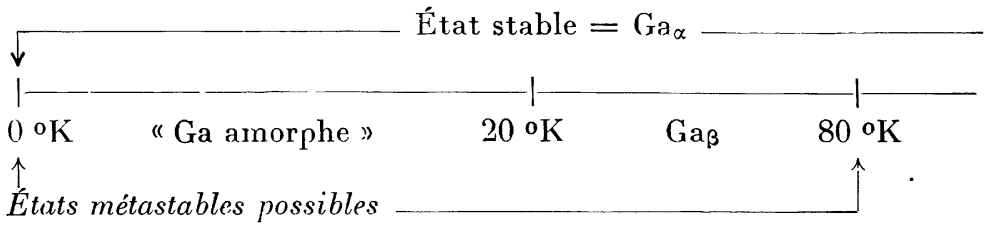

B) Cristallisation des gouttelettes liquides maintenues en surfusion

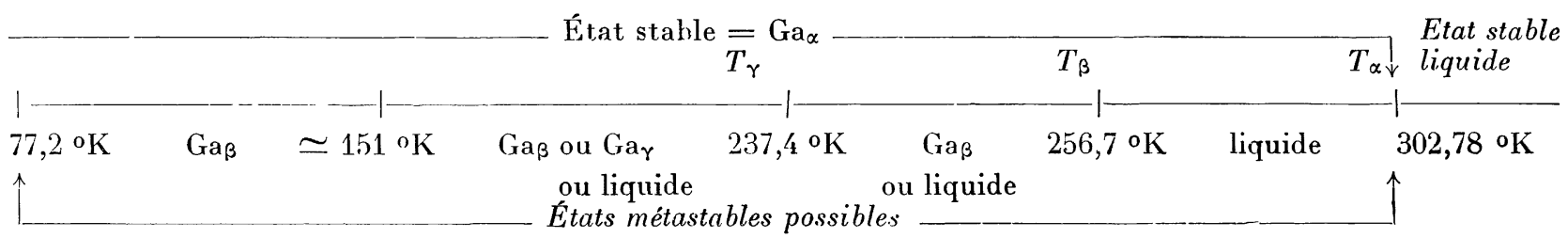

Sur la régle des degrés d'Ostwald. - A PARTIR DU liQuide. - Supposons qu'à une température donnée il puisse se former à partir d'un liquide maintenu en surfusion des phases $\gamma, \beta$ et $\alpha$ plus stables que la phase liquide initiale et que les potentiels chimiques de ces phases soient tels que $\mu_{1}>\mu_{\gamma}>\mu_{\beta}>\mu_{\alpha}$. La règle des degrés d'Ostwald énonce que le liquide cristallisera par degrés successifs en ces formes intermédiaires avant d'atteindre l'état stable final. Cependant, cette règle n'est pas toujours suivie et les conditions de l'apparition des phases métastables $\gamma$ et $\beta$ dépendent avant tout de la probabilité de formation des germes.

La vitesse de germination d'une phase $i$ est donnée par :

$$
J_{\mathrm{i}}=K \exp \left[-\frac{\Delta G^{*}}{k T}\right]=K \exp \left[-\frac{A_{\mathrm{i}} \sigma_{\mathrm{i}}^{\mathbf{3}}}{\rho_{\mathrm{i}}^{2} \Delta \mu_{\mathrm{i}}^{2}} \cdot \frac{1}{k T}\right]
$$

avec

$\Delta \mu_{\mathrm{i}}=\mu_{1}-\mu_{\mathrm{i}}: \mu_{\mathrm{i}}$ étant un potentiel chimique de la forme i,

$\rho_{i}$ : densité de la phase solide i qui apparaît,

$A_{\mathrm{i}}$ : est un facteur positif sensiblement identique pour les différentes phases d'une même substance,

$\sigma_{i}$ : est l'enthalpie libre interfaciale entre les phases en présence.

Il en résulte que la cristallisation du liquide en la forme métastable $\beta$ ou en la forme stable $\alpha$ dépend du signe de l'expression

$$
\Delta G_{\beta}^{*}-\Delta G_{\alpha}^{*}=\frac{\sigma_{\beta}^{3}}{\rho_{\beta}^{2} \Delta \mu_{\beta}^{2}}-\frac{\sigma_{\alpha}^{3}}{\rho_{\alpha}^{2} \Delta \mu_{\alpha}^{2}}
$$

ou encore de :

$$
f(T)=\frac{\rho_{\alpha} \Delta \mu_{\alpha}}{\sigma_{\alpha}^{3 / 2}}-\frac{\rho_{\beta} \Delta \mu_{\beta}}{\sigma_{\beta}^{3 / 2}} .
$$

Dans le cas du gallium, la variation des potentiels chimiques $\mu_{1}, \mu_{\beta}$ et $\mu_{\alpha}$ des phases liquides, Ga $\beta$ et $\mathrm{Ga}_{\alpha}$, en fonction de la température est représentée sur la figure 8.

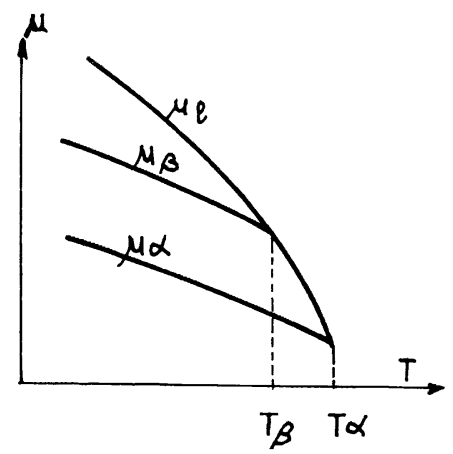

Fig. 8. - Allures du potentiel chimique des phases: liquide, $\mathrm{Ga}_{\beta}$ et $\mathrm{Ga}_{\alpha}$, en fonction de la température.

Lorsque la température est légèrement inférieure à $T_{\beta}=-16,3^{\circ} \mathrm{C}, \Delta \mu_{\beta} \simeq 0$ et $f(T)$ est positif ; il s'ensuit que $\Delta G_{\beta}^{*}$ est supérieur à $\Delta G_{\alpha}^{*}$ et $J_{\beta}<J_{\alpha}$ :

(1) Des mesures récentes effectuées en collaboration avec le laboratoire, C. N. R. S. " très basses températures" de Grenoble ont donné $6,0 \pm 01 \mathrm{oK}$ comme température critique d'une forme métastable à la pres sion atmosphérique. (C R. Acad. Sci. 1965, 261, 5431.) 
si le liquide cristallise, il le fera en la forme Ga $\alpha$. Si la température à laquelle le liquide peut se conserver est très inférieure à $T_{\beta}$ l'écart relatif entre les valeurs de $\Delta \mu_{\alpha}$ et $\Delta \mu_{\beta}$ diminue et l'influence des termes $\sigma$ sur le signe de $f(T)$ croît notablement. Nous avons vu que $\sigma_{\beta}$ a une valeur voisine de $\sigma_{\alpha} / 2$, donc $f(T)$ peut devenir négatif pour des températures très inférieures à $T_{\beta}$; dans ce cas, $J_{\beta}$ devient supérieur à $J_{\alpha}$ et le liquide cristallise en la forme $\mathrm{Ga}_{\beta}$. Il doit exister une température $T_{1}$ pour laquelle la condition $f(T)=0$ est satisfaite ; à cétte température $T_{1}$ le liquide doit cristalliser indifféremment en $\mathrm{Ga}_{\alpha}$ ou en $\mathrm{Ga}_{\beta}$ : l'expérience montre en effet que cette température $T_{1}$ se situe approximativement vers $-45^{\circ} \mathrm{C}$.

Dans le cas général, les variations de $\sigma_{\mathrm{i}}$ et de $\Delta \mu_{\mathrm{i}}$ avec la température ne sont pas connues mais il se peut que nous ayons la succession suivante :

$$
\begin{aligned}
& f(T)>0 \text { pour } T>T_{1} \\
& f(T)<0 \text { pour } T_{1}>T>T_{2} \\
& f(T)>0 \text { pour } T<T_{2} .
\end{aligned}
$$

Les températures $T_{1}$ et $T_{2}$ sont des racines de l'équation $f(T)=0$; par suite, la règle des degrés d'Ostwald ne s'applique que dans l'intervalle des températures comprises entre $T_{1}$ et $T_{2}$. Effectivement Gar n'apparaît qu'entre les températures $T_{1} \simeq-55^{\circ} \mathrm{C}$ et $T_{2} \simeq-100^{\circ} \mathrm{C}$. L'expression de $f(T)$ devient :

$$
f(T)=\frac{\rho_{\beta} \Delta \mu_{\beta}}{\left[\sigma_{\beta} g_{\beta}(\theta)\right]^{3 / 2}}-\frac{\rho_{\gamma} \Delta \varphi_{\gamma}}{\left[\sigma_{\gamma} g_{\gamma}(\theta)\right]^{3 / 2}}
$$

la signification des termes $g_{\beta}(\theta)$ et $g_{\gamma}(\theta)$ est celle donnée précédemment (formule 6 ).

A partir de la phase vapeur. - Palatnik et Zorine [44] ont montré qu'il convenait de tenir compte des termes du second degré dans le développement en série de Taylor de la variation du potentiel chimique $\Delta \mu_{\mathrm{i}}$ :

$$
\Delta \mu_{\mathrm{i}}=\frac{L_{\mathrm{i}}}{T_{\mathrm{i}}}\left(T_{\mathrm{i}}-T\right)-\frac{1}{2} \frac{\Delta C_{\mathrm{i}}}{T_{\mathrm{i}}}\left(T_{\mathrm{i}}-T\right)^{2} .
$$

$L_{\mathrm{i}}$ est la chaleur de transformation de la phase i en la phase vapeur à la température d'équilibre $T_{\mathbf{i}}$, $\Delta C_{\mathrm{i}}$ est la différence des capacités calorifiques à pression constante des formes considérées. Pour exprimer $f(T)$ (formule 12), on obtient ainsi une équation du second degré en $T$ et l'application ou la non-application de la règle des degrés d'Ostwald dépend de la discussion des racines $T_{1}$ et $T_{2}$ de l'équation $f(T)$. Une telle résolution nécessite la connaissance des capacités calorifiques et des enthal- pies libres interfaciales des différentes phases jusqu'aux très basses températures; néanmoins l'existence des températures limites $T_{1}$ et $T_{2}$ s'est vérifiée au cours d'un ensemble d'études concernant la condensation des vapeurs de seize métaux $(\mathrm{Pb}$, Sn, Bi, In, ...) ; ces études ont permis à Palatnik [45] d'établir expérimentalement que la règle des degrés s'applique alors dans tout l'intervalle de température extérieur aux racines $T_{1}$ et $T_{2}$ selon le diagramme suivant :

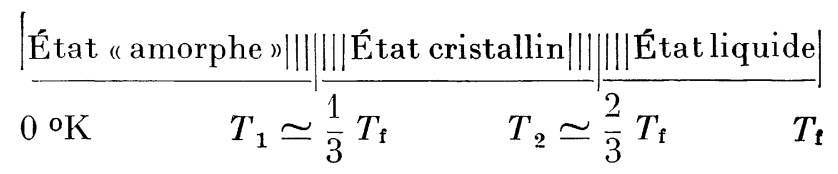

Les zones hachurées délimitent les intervalles où l'apparition de l'une quelconque des phases adjacentes est possible. Par condensation de la vapeur de gallium, Bückel et al. [43] montrent bien l'existence d'une température $T_{1}$ délimitant deux domaines dans lesquels le gallium se trouve soit à l'état amorphe soit à l'état cristallin.

D'autre part, par échauffement du film amorphe, celui-ci se transforme vers $20{ }^{\circ} \mathrm{K}$ en $\mathrm{Ga} \beta$, la règle des degrés s'applique encore à cette température. Signalons toutefois qu'à partir de ce film amorphe ces auteurs n'ont obtenu qu'une seule forme cristal. line métastable.

Les résultats concernant le polymorphisme du gallium à la pression atmosphérique montrent l'importance du choix de très petits échantillons dans l'étude des changements de phase, et aussi de leur protection contre les agents extérieurs. L'obtention de gouttelettes liquides très pures enrobées d'un film protecteur doit permettre de réaliser les conditions de la germination homogène et d'observer les types possibles de cristallisation. Si l'intervalle de température dans lequel la substance reste en surfusion est suffisamment étalé il doit être possible d'observer la cristallisation du liquide en formes métastables correspondant ̀̀ des états stables à haute pression. Dans cette recherche, le cas du gallium a été favorable par suite de la structure complexe de la forme stable à la pression atmosphérique : il peut ainsi apparaître des formes intermédiaires dont l'énergie de formation du germe est plus faible. Par condensation de la vapeur en couches minces, il est aussi possible d'obtenir des phases cristallines métastables (condensation en $\mathrm{Ga} \beta$ par exemple) puisqu'à partir d'un état désordonné toutes les possibilités de cristallisation sont permises. La réalisation d'un ultravide et la condensation sur des supports débarrassés d'impuretés adsorbées doivent permettre des progrès dans cette étude.

Manuscrit reçu le 28 juillet 1965. 


\section{BIBLIOGRAPHIE}

[1] Bosro (L.), Thèse d'État, Paris, 1965, Mét. Corr. Ind. 1965, no 483, 421 et $\mathrm{n}^{\circ} 484,451$.

[2] Vormer (M.), Kinetik der Phasenbilitung, Leipzig, Steinkopf, 1939.

[3] Frankel (J.), Théorie cinétique des liquides, Dover Public., New York, 1955.

[4] Tammann (G.), Agregatzustände, Leipzig, 1929.

[5] Turnbula (D.), J. Chem. Physics, 1952. 20, 412.

[6] Defrain (A.), Thrise d'État, Paris ; Mét. Corr. Ind., 1960, no 417, 175.

[7] Davilov (V. I.), Structure et cristallisation des liquides, Acad. U. R. S. S., 1956.

[8] Defrain (A.), Mét. Corr. Ind., 1962, no /138, 1.

[9] Bosio (L.), Defrain (A.) et Epelboin (I.), $C . R$. Acad. Sc., Paris, 1961, 253, 2343.

[10] Defrain (A.) et Frelboin (I.), J. Physique Rad., $1960,21,76$.

[11] Delcroix (S.), Defrain (A.) et Epelboin (I.), J. Physique Rad., 1963, 24, 17.

[12] Turniull(D.), J. Chem. Physics, 1950, 18, 198.

[13] Bosio (L.) et Defrain (A.), C.R. Acad. Sc., Paris, 1962, 254, 1020.

[14] Bosio (L.) et Defrain (A.), C. R. Acad. Sc., 1963, 257, 901 .

[15] Hollomon (J.) et Turniull (D.), Progress in metal physics, Pergamon Press, Lundres, 1953, 4.

[16] Buckle (F. R.), Proc. Poy. Soc., 1961, 261, 190.

[17] Tolman (R. C.), J. Chem. Physics, 1949, 17, 118.

[18] Kirkw ood et Buff, J. Chem. Physics, 1949, 17, 338.

[19] Tunnulu. (D.), Solid State Physics, Acad. Press, New York, 1956, 3, 225.

[20] Bosio (L.), Defrain (A.) et Erpelboin (I.), J. Physiqrie Rad., 1962, 23, 878.

[21] Andarelli (G.), Diplôme d'Études Suṕrieures, Paris, 1965.

[22] Bosio (L.) et Defrain (A.), C. R. Acad. Sc., 1964, 258, 4929.

[23] Defrain (A.), C. R. Acad. Sc., 1960, ?50, 483.

[24] Kaufman (L.), Solids under Pressurr, MacGrawHill, 1963, p. 377 .
[25] Bosio (L.), C. R. Acad. Sc., 1964, 259, 4545.

[26] Blanconnier (P.), Bosio (I.), Defrain (A.), Rrmsky (A.) et C.urien (H.), Bull. Soc. Franç. Minér. Crist., 1965, 88, 145.

[27] Curien (H.), Rimsky (A.) et Defrain (A.), Bull. Soc. Franc. Minér. Crist., 1961, 84, 260.

[28] Laves (F.), Z. Krist., 1933, 84, 256.

[29] Brahama, Sharma (I.) et Donohue (J.), Z. Krist., 1962, 117, 293.

[30] Richlinds (T. W.) et Boyer (S.), J. Amer. Chem. Soc., 1921, 43, 280.

[31] Adams (C. B.), Johnston (H. L.) et Kerr (E. C.), J. Amer. Chem. Soc., 1952, 74, 4784.

[32] ERry (M.), Thèse d'État, Paris, 1958, Éditions Métaux.

[33] Bosio (L.), Defrain (A.) et Epelboin (I.), C. $R$. Acad. Sc., 1960, 250, 2553.

[34] Menke (H.), Physik Z., 1932, 33, 593.

[35] Hendus (H.), Z. Naturf., 1947, 2a, 505.

[36] Bertie (J. E.), Calvert (L. D.) et Whaliey (E.), Canad. J. Chem., 1964, 42, no 6, 1373-1378.

[37] Bridgman (P. W.), Phys. Rev., 1935, 48, 893.

[38] Jayaraman (A.), Krement (W.), Newton (R. C.) et Krnnedy (G. C.), J. Phys. Chem. Solids, 1963, 24, 7 .

[39] Voronel (A. V.), Z. Tekh. Fiz., 1958, 28, no 11, 2630.

[40] Simon (F. E.) et Glatzel (G.), Z. Anorg. Allgem. Chem., 1929, 178, 309.

[41] Gilvariy (J. J.), Phys. Rev., 1956, 102, 308.

42] Zoкнovsкi (M. K.), Z. Fiz. Khim., 1963, 37, 2635.

[43] Bǘckel (W.) et Gey (W.), Z. Physik, 1963, 176, 336.

[44] Palatnik (I. S.) et Zorine (V. S.), Z. Fiz. Khim., 1959, 33, 1859.

[45] Palaticik (L. S.) et Papirov (I. I.), Croissance orientée, Éd. "Métallurgie ", Moscou, 1964.

Note ajoutée sur épreuves. - La susceptibilité paramagnétique de $\mathrm{Ga} \beta$ à $17{ }^{\circ} \mathrm{C}$ est environ douze fois plus élevée que celle du gallium liquide (mesures en cours de M. Jamet). 